\section{Transition probabilities and the incidence of filled pauses}

\author{
MARK COOK, University of Aberdeen, Old \\ Aberdeen, Scotland
}

Maclay \& Osgood's theory about filled pauses (FPs) is described and evidence relevant to it summarized. Maclay \& Osgood's own evidence for their hypothesis is not very satisfactory; suggestions are made for a better method of testing the hypothesis, using the "cloze" method to estimate transition probabilities. The results show that words following FPs have a lower transition probability than other words, with the exception of pronouns where there is no difference. The significance of this finding is discussed in the light of Maclay \& Osgood's theory.

Filled pauses-er, um, and variants-occur with monotonous regularity in most people's speech; their function, if any, is still uncertain. It was originally thought (Mahl, 1956) that they were a result of anxiety, but subsequent work (Kasl \& Mahl, 1965;Cook, 1969) has shown that this is not so. Maclay \& Osgood (1959) have put forward a different theory; they suggest that the FP is the speaker's reaction to his own silence. He makes this response if he thinks that someone else might break in and "take the floor." In other words, the FP is a signal by the speaker that he has not finished even though he is temporarily silent. According to this hypothesis, FPs are produced when (1) the speaker "dries up" and (2) is afraid of interruption during this silence. That is, if the speaker is more liable to "dry up" because his material is more difficult and/or if he feels more liable to interruption, his rate of FPs will increase. Either or both circumstance(s) will lead to an increase in FPs.

Most attempts to prove the hypothesis have concentrated on the first factoruncertainty or difficulty. It should be noted, however, that evidence that FPs occur at points of uncertainty is also consistent with the hypothesis that FPs are merely an alternative and equivalent form of the unfilled pause. Moreover, even the evidence that FPs occur when the speaker is thinking what to say is not very conclusive. For example, Maclay and Osgood's evidence is not very satisfactory; they reported that FPs were more likely to occur before some types of words than before others. However, they did not examine every FP in a certain sample of speech but only those that occurred in certain selected and supposedly representative grammatical sequences; unpublished work by the author, using a better method, has not replicated their findings. Other work on FPs and difficulty is equally inconclusive. It is well established (Goldman-Eisler, 1968) that unfilled pauses occur at points of difficulty; however, Boomer (1965) found that a large proportion of FPs did not occur during long unfilled pauses, which suggests that FPs could be unrelated to difficulty. Goldman-Eisler found that FPs did not increase while speakers were summarizing the point of a cartoon sequence, as opposed to simply describing it, whereas unfilled pauses did increase during the more difficult task of summary. However, Paivio (1965), who used a larger number of Ss, did find an increase in FPs in the summary condition. Evidently it is by no means certain that FPs occur at points of difficulty and some further investigation is needed.

Maclay and Osgood's method was a crude one; they assumed that "lexical" wordsroughly speaking, nouns, verbs, and adjectives-are more difficult than other words. This is probably true overall, but obviously a more sensitive method of estimating the difficulty of single words would be better. One possible method is the estimation of transition probability, i.e., the probability of the word occurring, given what has gone before. This probability is usually estimated by the method of successive guesses, the so-called "cloze" method (Goldman-Eisler, 1968). A number of Ss in turn try to guess each successive word in the sentence, and the ratio of correct guesses to total guesses is taken as the estimate of transition probability. The lower the ratio the lower the probability, and the more difficult the word is. Therefore, if filled pauses do occur at points of high difficulty, they should occur before words of low transition probability.

It may be objected that this hypothesis involves a very naive view of language. Speakers do not select each word separately on the basis of what has gone before. The process of producing speech is more complex; in particular, the speaker must organize his grammar. It is therefore very artificial to estimate the "difficulty" of words in a sentence by the transition probability method. Obviously, it is true that language production is more complex than this model assumes, but nevertheless, until some more is known about the processes involved, no better method seems available. The transition probability method can be used to examine variations in difficulty of individual words within the
Table 1

Number of Words Preceded and Not Preceded by FPs

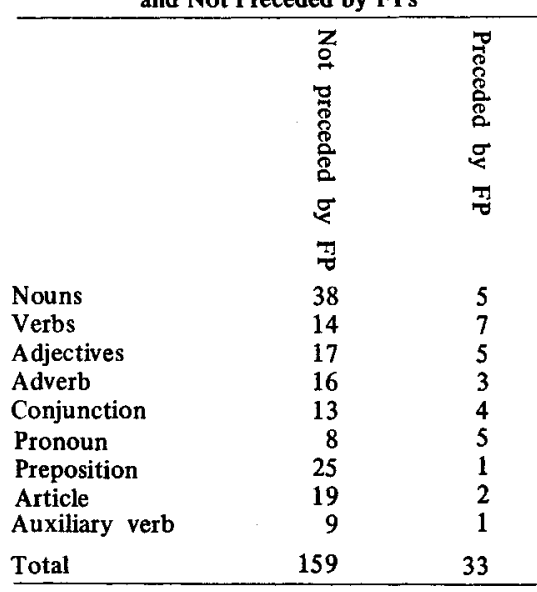

grammatical structure; for example, one noun, which is the subject of a clause, may be more difficult than another that is also the subject of a sentence. In any case, the method has been used effectively by Goldman-Eisler on similar material.

\section{METHOD}

Fourteen sentences were taken from interview data. They were selected according to two criteria. (1) The sentences were reasonably coherent and logical, free from sudden changes of direction in grammar and meaning. This criterion was also used by Goldman-Eisler (1968). (2) The sentences contained FPs preceding various parts of speech, as shown in Table 1. Table 1 also gives the total number of occurrences of each part of speech not preceded by FPs.

Five Ss then guessed each word of every sentence successively. They were given a general indication of the subject of the sentence and the first two words, and then tried to guess the third word. They were allowed up to $1 \mathrm{~min}$ to guess, and, if they had not guessed it by then, they were told it. Every incorrect suggestion made by the first $S$ was noted, and every new incorrect suggestion made by subsequent $\mathrm{Ss}$ was also noted.

\section{RESULTS}

Ratios of number of correct guesses to total number of possibilities suggested were calculated for each word. These ratios range from 0 , where no $S$ guessed the correct word, to 1.00 where all five Ss guessed the correct word immediately. These ratios are the estimates of the transition probabilities. The ratios were all transformed to arcsin scores for the statistical analysis.

The mean ratio for the 159 words not preceded by FPs was significantly higher than the ratio for the 33 words preceded by FPs $(t=3.89, p<.001)$

Table 2 shows the ratios, for words preceded by FPs and words not preceded by FPs, for each part of speech separately. It 
Table 2

Mean Transition Probability of Words Preceded by FPs and of Words Not Preceded by FPs

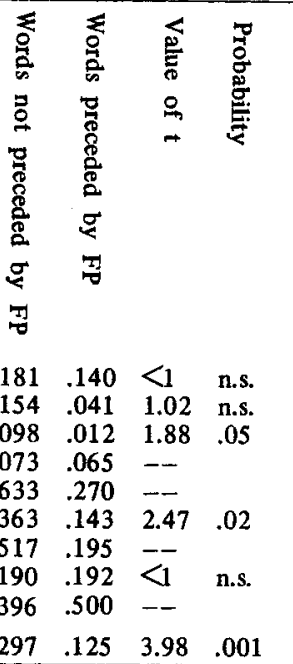

shows that the transition probability of words following FPs was lower for every part of speech except pronouns and auxiliary verbs, although the difference is not significant in every case. ( $t$ values could not be calculated in some cases, because the numbers were not large enough.)

\section{DISCUSSION}

The hypothesis has been confirmed; there is a relation between transition probability and incidence of FPs. Words preceded by FPs have a transition probability less than half that of other words. Furthermore, the relation holds for each part of speech separately, so it cannot be an artifact caused by the inclusion of some very "easy" words in the sample of words not preceded by FPs.

The only part of speech for which the relation does not hold is the pronoun. (There was only one auxiliary verb preceded by an FP in the sample.) This is interesting, inasmuch as the author has found, in unpublished research, that FPs occur significantly more often before pronouns and conjunctions than before other parts of speech. FPs that occur before pronouns and conjunctions tend to come at the beginning of a pronoun-verb sequence, i.e., at the beginning of a clause. This seemed to indicate that they occurred while the speaker was organizing his clause. Boomer (1965) reached similar conclusions on different evidence. It is particularly interesting, therefore, that the occurrence of an FP before such pronouns is apparently unrelated to the difficulty of that pronoun. This is exactly what would be expected, if indeed the speaker is pausing to organize his grammar, and not to "find" the pronoun. The relation does not hold for conjunctions, however.

The data in Table 2 also give some support for Maclay and Osgood's assump- tion that nouns, verbs, and adjectives are more "difficult" than other parts of speech, since their transition probabilities are generally lower than those of other parts of speech. Note, however, that adverbs and pronouns also have low probabilities; evidently Maclay and Osgood's method was rather crude and indiscriminating.

This study has examined the occurrence of FPs at one type of decision point, namely where the speaker is looking for a particular difficult word. It cannot be taken to prove that FPs occur at all points of difficulty, for it is quite possible they do not occur so of ten at points of grammatical or semantic decision. (Indeed, FPs do seem more frequent where the speaker is going through a list, a task that involves only finding individual words and requires no grammatical decisions.) Furthermore, there almost certainly are other ways of indicating noncompletion and continuing to produce sounds while having nothing to say, e.g., repetition or the use of various more or less meaningless words and phrases, such as "you know" or "I mean." Now that it has been established that FPs do occur at some points of difficulty, a more extensive study is required that examines the incidence of FPs in relation to individual difficult words, grammatical complexity, and difficulty of topic, and that also examines incidence of possible equivalents of FPs.
Future work should also look for a different way of estimating difficulty of individual words, since the "cloze" method is exceedingly laborious. Possibly a quicker way of estimating transitional probabilities could be found; alternatively, word frequency lists could be used.

To conclude, it does appear that FPs are related to difficulty of selection of individual words, so that the first part of Maclay and Osgood's hypothesis is at least tenable. However, much more work needs to be done to specify more precisely when FPs and their equivalents occur.

\section{REFERENCES}

BOOMER, D. S. Hesitation and grammatical encoding. Language \& Speech, 1965, 8, 148-158.

COOK, M. Anxiety, speech disturbance and speech rate. British Journal of Social \& Clinical Psychology, 1969, 8, 13-21.

GOLDMAN-EISLER, F. Psycholinguistics: Experiments in spontaneous speech. London: Academic Press, 1968.

KASL, S. V., \& MAHL, G. F. The relationship of disturbances and hesitations in spontaneous speech to anxiety. Journal of Personality \& Social Psychology, 1965, 1, 425-433.

MACLAY, H., \& OSGOOD, C. E. Hesitation phenomena in spontaneous English speech. Word, 1959, 15, 19-44.

MAHL, G. F. Disturbances and silences in the patient's speech in psychotherapy. Journal of Abnormal \& Social Psychology, 1956, 53, 1-15.

\section{Recognition memory of preschool children for pictures and words ${ }^{1}$}

DAVID A. CORSINI, KENNETH A.
JACOBUS, and S. DAVID LEONARD,
University of Georgia, Athens, Ga. 30601

Preshool children's memory of words and pictures was examined using a recognition-memory task. The results indicated that pictures were better retained than words. These findings were discussed in terms of previous research and theory that has dealt with children's modes of representing information.

Bruner (1964) has suggested that there are three ways by which the human organism extracts meaning from his environmental experience or represents his experience. These three means are: (a) representation on the basis of physical actions (enactive); (b) representation by imagery (ikonic); and (c) representation by symbols (symbolic). While the adult is capable of using all three means of representation, the ability to use all three is not present from birth. Bruner suggests that the preschool child is capable of using both enactive and ikonic means of representation but has only rudimentary ability to use symbolic representation. If this were correct, one consequence might be that the preschool child's capacity for storing information in memory would be better for pictures than for words.

There have been two studies with young children which at first glance appear to contradict this expectation. Ducharme \& Fraisse (1965) found that the free recall of children was less for pictures than for their concrete noun labels. Dilley \& Paivio (1968), comparing children's learning in a paired-associate task where either pictures or words were used as the response elements, found that learning was more rapid with words than with pictures.

There are at least two interpretations of these results. First, it is possible that children have a poorer capacity for storing information presented pictorially than verbally. This interpretation would run counter to Bruner's position. Second, it is 